\title{
EFEITOS DE UMA INTERVENÇÃO COM PEDÔMETRO SOBRE O RISCO METABÓLICO EM CRIANÇAS OBESAS
}

EFFECTS OF AN INTERVENTION WITH PEDOMETER ON METABOLIC RISK IN OBESE CHILDREN

EFECTOS DE UNA INTERVENCIÓN CON PODÓMETRO EN EL RIESGO METABÓLICO EN NIÑOS OBESOS

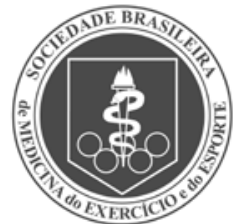

Artigo Original

Original ARtICle Artículo Original
Antonio Stabelini Neto

(Educador Físico)

Renan Camargo Corrêa ${ }^{1}$

(Educador Físico)

João Paulo de Farias ${ }^{1}$

(Educador Físico)

Géssica Castilho dos Santos ${ }^{1}$

(Educadora Física)

Claudinei Ferreira dos Santos ${ }^{1}$

(Educador Físico)

Rui Gonçalves Marques Elias'

(Educador Físico)

Waynne Ferreira de Faria

(Educador Físico)

1. Universidade Estadual do Norte do Paraná, Jacarezinho, PR, Brasil.

\section{Correspondência:}

Universidade Estadual do Norte do Paraná (UENP). Al. Padre Magno, 841, Jacarezinho, PR, Brasil. 86400-000.

asneto@uenp.edu.br

\section{RESUMO}

Introdução: Nas últimas décadas, a incidência de obesidade na população pediátrica vem aumentando, juntamente com a inatividade física. A obesidade e a inatividade física são componentes predisponentes para o agravo dos fatores de risco metabólico, sendo de fundamental importância seu tratamento visando melhora da saúde dos jovens. Objetivo: Verificar o efeito de 12 semanas de uma intervenção focada na atividade física sobre os fatores de risco metabólico em crianças obesas. Métodos: A amostra final foi composta por 19 escolares com idade entre oito e 10 anos (10 no grupo pedômetro e nove no grupo controle). A atividade física foi mensurada e orientada por meio do pedômetro Yamax ${ }^{\circledR}$ sw 700 (Tóquio, Honchu, Japão). Para comparações intra e entre grupos foi aplicado a ANOVA two way para medidas repetidas com significância estipulada em $p<0,05$. Resultados: O número de passos aumentou significativamente somente no grupo pedômetro após as 12 semanas de intervenção (pré $9729,7 \pm 2539,8$ vs. pós $13151,6 \pm 3581,7)$. Foi observado aumento significativo do peso corporal, da estatura e do IMC no grupo controle. No grupo pedômetro, observou-se aumento significativo apenas na estatura. Com relação aos fatores de risco metabólicos, não foi encontrada mudança significativa no grupo controle, enquanto no grupo pedômetro foi observada diminuição significante nos valores de insulina (pré 12,21 $\pm 8,53$ vs. pós 7,26 $\pm 4,70 \mu \mathrm{Ul} / \mathrm{ml}$ ) e HOMA IR (pré 2,76 $\pm 1,93$ vs. pós 1,49 $\pm 0,93$ ). Conclusão: A presente intervenção com atividade física a partir do estabelecimento de metas utilizando pedômetros reduziu significativamente as concentrações de insulina e o índice de homeostase (HOMA IR) em crianças obesas, independentemente da perda de peso.

Descritores: obesidade; atividade motora; pediatria.

\begin{abstract}
Introduction: In recent decades, the incidence of obesity in children is increasing along with physical inactivity. Obesity and physical inactivity are predisposing components to the worsening of metabolic risk factors and treatment is fundamental aiming at improving the health of young people. Objective: To verify the effect of 12 weeks of an intervention focused on physical activity on metabolic risk factors in obese children. Methods: The final sample consisted of 19 students aged between 8 and 10 years (10 in the pedometer group and nine in the control group). Physical activity was measured and guided by the Yamax ${ }^{\oplus}$ sw 700 pedometer (Tokyo, Honshu, Japan). For within and between groups comparisons the two way ANOVA was applied for repeated measures with significance set at $p<0.05$. Results: The number of steps increased significantly only in the pedometer group after the 12-week intervention ( $9729.7 \pm 2539.8$ before vs. 13151.6 \pm 3581.7 after). Significant increase was observed in body weight, height, and BMI in the control group. In the pedometer group, it was observed significant increase only in stature. Regarding the metabolic risk factors, there was no significant change found in the control group, while in the pedometer group a significant decrease in insulin levels was observed (12.21 \pm 8.53 before vs. $7.26 \pm 4.70 \mu \mathrm{UI} / \mathrm{ml}$ after) and HOMA IR (2.76 \pm 1.93 beforevs. $1.49 \pm 0.93$ after). Conclusion: This intervention with physical activity from setting goals using pedometers significantly reduced the concentrations of insulin and the homeostasis index (HOMA IR) in obese children regardless of weight loss.
\end{abstract}

Keywords: obesity; motor activity; pediatrics.

\section{RESUMEN}

Introducción: En las últimas décadas, la incidencia de la obesidad en los niños es cada vez mayor, junto con la inactividad física. La obesidad y la inactividad física son componentes que predisponen al empeoramiento de los factores de riesgo metabólico, siendo de fundamental importancia el tratamiento destinado a mejorar la salud de los jóvenes. Objetivo: Comprobar el efecto de 12 semanas de una intervención centrada en la actividad física sobre los factores de riesgo metabólico en los niños obesos. Métodos: La muestra final fue de 19 estudiantes de edades comprendidas entre ocho y 10 años (10 en el grupo podómetro y nueve en el grupo de control). La actividad física se midió y fue guiada por el podómetro Yamax ${ }^{\circledR}$ sw 700 (Tokio, Honchu, Japón). Para las comparaciones dentro y entre grupos se aplicó la ANOVA de dos vías para medidas repetidas, con una significación establecida en $p<0,05$. Resultados: El número de pasos aumentó significativamente sólo en el grupo podómetro después de 12 semanas de intervención (pre 9729,7 $\pm 2539,8$ vs. post 13151,6 2 3581,7). Se observó un aumento significativo en el peso corporal, la estatura y el IMC en el grupo control. En el grupo podómetro, hubo un aumento significativo sólo en estatura. En cuanto a los factores de riesgo metabólico, no hubo ningún cambio significativo en el grupo control, mientras que en el grupo podómetro se observó disminución significativa de los valores de insulina (pre 12,21 $\pm 8,53 \mathrm{vs}$. post 7,26 $\pm 4,70 \mu \mathrm{Ul} / \mathrm{ml}$ ) y HOMA IR (pre 2,76 $\pm 1,93$ vs. post 1,49 $\pm 0,93$ ). Conclusión: Esta intervención con actividad 
física a partir del establecimiento de metas utilizando podómetros redujo significativamente las concentraciones de insulina y el índice de la homeostasis (HOMA-IR) en niños obesos, independientemente de la pérdida de peso.

\section{Descriptores: obesidad; actividad motora; pediatría.}

\section{INTRODUÇÃO}

A obesidade é uma doença multifatorial que está associada ao desenvolvimento de inúmeras doenças crônicas não transmissíveis como diabetes mellitus, dislipidemia e hipertensão arterial1,2. Dados recentes apontam um crescente aumento na incidência de obesos na população mundial ${ }^{2-4}$. Embora suas causas sejam multifacetadas, a inatividade física tem sido apontada como um dos principais fatores relacionados a obesidade, uma vez que interfere diretamente sobre o balanço energético ${ }^{5}$.

Considerando que aproximadamente $70 \%$ das mortes prematuras de adultos estão relacionadas a comportamentos inadequados que se iniciaram durante a infância e adolescência ${ }^{6}$, tem sido descrito que a manutenção de níveis adequados de atividade física na infância está relacionada a um estilo de vida ativo na idade adulta que consequentemente pode contribuir para um melhor perfil metabólico e menores taxas de morbimortalidade por doenças crônicas não transmissíveis ${ }^{7-9}$.

Nessa perspectiva, a World Health Organization ${ }^{10}$ sugere que crianças e adolescentes devem se engajar em atividades físicas de intensidade moderada a vigorosa (AFMV) por pelo menos 60 minutos por dia. Entretanto, diversos estudos apontam alta prevalência de inatividade física em crianças e adolescentes brasileiros ${ }^{11,12}$. Dessa forma, programas de promoção à saúde devem ser desenvolvidos com crianças e adolescentes com o intuito de aumentar a conscientização e adoção de hábitos saudáveis.

Nesse contexto, o monitoramento da quantidade de passos realizados diariamente e estabelecimento de metas utilizando pedômetros têm se apresentado uma estratégia viável para o aumento da prática de atividades físicas ${ }^{13,14}$. Uma revisão sistemática realizada por Bravata et al. ${ }^{15}$ sugerem que o uso de pedômetros estão associados à aumentos significativos de atividade física e diminuição do índice de massa corporal.

Adicionalmente, Manley et al., ${ }^{16}$ verificaram que uma intervenção utilizando pedômetros em adolescentes de 11 a 13 anos, foi eficaz para a melhora da aptidão aeróbica e nível de atividade física. Entretanto, apesar de ser evidenciado na literatura a eficácia do pedômetro sobre a prática de atividade física de crianças e adolescentes, ainda não está claro se uma intervenção com pedômetro de um período relativamente curto seria eficaz na modulação do perfil metabólico de crianças obesas. Portanto, o objetivo do presente estudo foi verificar o efeito de 12 semanas de uma intervenção focada na atividade física a partir do estabelecimento de metas utilizando pedômetros sobre os fatores de risco metabólicos de crianças obesas.

\section{MATERIAIS E MÉTODOS}

A população foi formada por crianças obesas com idade entre oito e 10 anos da rede municipal de ensino da cidade de Jacarezinho, PR, Brasil. Os critérios de inclusão para a participação do estudo foram: ter idade entre oito e 10 anos; ser classificado como obeso (a) de acordo com os critérios para idade e gênero do Centers for Desease Control and Prevention ${ }^{17}$; estar matriculado na rede municipal de ensino; apresentar o Termo de Consentimento Livre e Esclarecido assinado pelos pais ou responsáveis; e não apresentar algum tipo de doença metabólica reconhecida.

Inicialmente foram recrutadas 60 crianças de ambos os gêneros, sendo que 20 crianças não atenderam os critérios de elegibilidade, assim 40 foram designadas de forma aleatória simples para o grupo pedômetro $(n=20)$ e grupo controle $(n=20)$. Entretanto, durante o desenvolvimento da intervenção, ocorreram perdas amostrais em ambos os grupos (Figura 1).
Dessa forma, a amostra final contou com 19 crianças, sendo 10 do grupo pedômetro (3 meninas e 7 meninos) e nove do grupo controle (4 meninas e 5 meninos). O protocolo experimental foi aprovado pelo Comitê de Ética em pesquisa da Universidade Estadual do Norte do Paraná, PR, Brasil, sob o parecer 060/2012.

\section{Mensuração e orientação da atividade física}

Foram utilizados pedômetros da marca Yamax sw 700 (Tóquio, Honchu, Japão) para a análise inicial do número de passos e posteriormente para a orientação da atividade física. Cada criança utilizou um pedômetro na cintura ao longo do dia, exceto para dormir, nadar e tomar banho. Todas as noites os participantes foram orientados a anotarem o número de passos acumulados durante o dia em um formulário de registro, no qual foi enviado junto com o aparelho, e após a anotação, deveriam reiniciar o pedômetro. As crianças do grupo intervenção com pedômetro foram instruídas a realizar 30\% a mais de passos em relação à média de passos da linha de base e a caminhar e/ou brincar em um ritmo moderado. Para facilitar o cumprimento das metas e incentivá-las a aumentar a prática de atividade física, foram entregues folhetos com várias sugestões de atividades como: caminhada, basquetebol, futebol, voleibol, queimada, entre outras brincadeiras, com suas respectivas estimativas de passos a cada 15 min.

Essa intervenção focada na atividade física utilizando pedômetro baseia-se no pressuposto que a atividade física moderada por $10 \mathrm{~min}$ realiza 1000 passos $^{18}$, portanto, 6000 passos extras equivalem a aproximadamente 60 min de atividade física moderada, que por sua vez atende a recomendação de volume diário de atividade física para a população pediátrica ${ }^{10}$ A meta individualizada foi proposta a fim de estabelecer metas realistas com intuito de atingir pelo menos 15000 e 12000 passos/ dia nos meninos e meninas, respectivamente ${ }^{18}$. Todas as crianças foram orientadas a manter seus hábitos nutricionais durante o período do estudo.

\section{Instrumentos e Procedimentos}

A massa corporal foi mensurada por meio de uma balança digital (Welmy ${ }^{\circledR}$, Santa Bárbara do Oeste, São Paulo, Brasil), com precisão de 100 gramas e a estatura através de um estadiômetro portátil (Welmy ${ }^{\circledR}$, Santa Bárbara do Oeste, São Paulo, Brasil), fixado a parede, graduado de 0 a

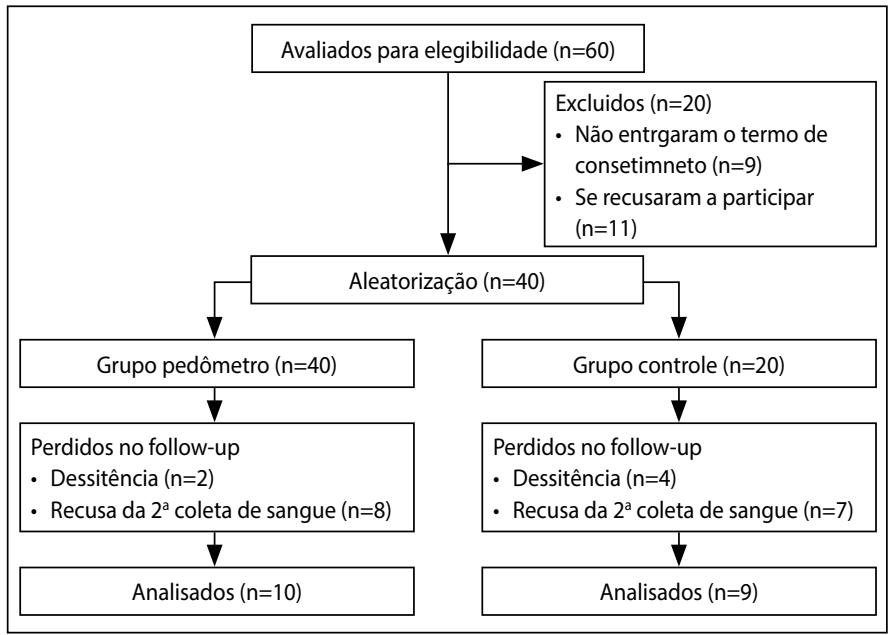

Figura 1. Delineamento do processo amostral. 
$200 \mathrm{~cm}$, com escala de precisão 0,1 cm. O índice de massa corporal (IMC) foi calculado por meio do quociente da massa corporal em quilogramas, pela estatura em metros quadrados. A circunferência da cintura (CC) foi aferida por uma fita métrica (Sanny ${ }^{\circledast}$, São Bernardo do Campo, São Paulo, Brasil) no ponto intermédio entre a última costela flutuante e a crista ilíaca. Para aferição dos valores de pressão arterial sistólica (PAS) e diastólica (PAD) foram utilizados um esfigmomanômetro aneroide e estetoscópio (Premium ${ }^{\oplus}$, Barueri, São Paulo, Brasil) com dimensões apropriadas para a faixa etária, no braço esquerdo de cada criança na posição sentada e após cinco minutos de repouso. Foram tomadas duas medidas com intervalo em torno de um minuto entre elas, conforme recomenda as VI Diretrizes Brasileiras de Hipertensão ${ }^{19}$.

A análise sanguínea foi realizada em um laboratório certificado, no qual as crianças foram encaminhadas em companhia dos seus responsáveis com uma guia de liberação do exame. Foram coletados aproximadamente $5 \mathrm{ml}$ de sangue da prega do cotovelo, no período da manhã (7:00 - 9:00 h) após $12 \mathrm{~h}$ de jejum, antes e após a intervenção para as análises de colesterol total (CT), triglicérides, lipoproteínas de alta densidade (HDL-C), glicose e insulina. Para o cálculo do índice de homeostase (HOMA-IR), foi necessário converter para mmol/L os valores de glicose obtidos em mg/dL. Para tanto, esses valores foram multiplicados por 0,0556. Pôde-se, então, calcular o HOMA-IR pela seguinte equação matemática: insulina x glicemia/22, $5^{20}$.

\section{Análise estatística}

Os dados estão apresentados em média (desvio padrão) e foram analisados no programa estatístico SPSS (Armonk, Nova lorque, Estados Unidos), versão 22.0. ANOVA para medidas repetidas com dois fatores foi empregada para determinar diferenças significativas entre os respectivos grupos (pedômetro vs controle) e entre os momentos (pré vs pós). Caso o teste de esferecidade de Mauchly fosse violado, a correção de Greenhouse-Geisser foi assumida. Quando o teste F identificou efeito e/ou interação o post hoc de Bonferroni foi aplicado para localizar as diferenças entre as médias. Para complementar as análises o tamanho do efeito foi apresentado $\left(n^{2}\right)$. A significância foi estipulada em $5 \%(p<0,05)$.

\section{RESULTADOS}

A Figura 2 apresenta a média do número de passos/dia em ambos os grupos nos momentos pré e pós-intervenção. Houve efeito do momento $\times$ grupo $\left(F=29,344 ; p<0,001 ; \eta^{2}=0,633\right.$ ) no número de passos/ dia. Assim, nota-se que após 12 semanas o grupo pedômetro aumentou significativamente a média do número de passos/dia em relação ao momento pré e em comparação ao momento pós do grupo controle.

As características antropométricas de ambos os grupos nos momentos pré e pós-intervenção estão apresentadas na Tabela 1. Observa-se que a estatura aumentou significativamente após 12 semanas em ambos os grupos. Não houve mudança significativa para as demais medidas antropométricas avaliadas no grupo pedômetro. Já no grupo controle, a massa corporal e IMC aumentaram significativamente durante o período de intervenção.

A Tabela 2 apresenta os resultados dos fatores de risco metabólicos nos momentos pré e pós-intervenção de atividade física com pedômetro em crianças obesas. Após 12 semanas, houve efeito do momento nos valores de insulina $\left(F=5,323 ; p=0,034 ; \eta^{2}=0,238\right)$ e HOMA-IR ( $\left.F=5,977 ; p=0,026 ; \eta^{2}=0,260\right)$. Desta forma, observa-se que somente as concentrações de insulina e o HOMA IR reduziram significativamente no grupo pedômetro. Quanto ao grupo controle, as variáveis analisadas não apresentaram significância estatística.

\section{DISCUSSÃO}

Ao longo das últimas décadas, tem se dado ênfase para a promoção de um estilo de vida ativo a partir de atividades físicas de intensidade moderada que podem ser realizadas ao longo do dia. Nesta perspectiva, o pedômetro pode ser utilizado como ferramenta para estimular a prática de atividades físicas em intervenções que visam a prevenção e/ou tratamento de alguns fatores de risco para as doenças crônicas não transmissíveis ${ }^{1,21,22}$.

O presente estudo avaliou o efeito de uma intervenção focada na atividade física a partir do estabelecimento de metas utilizando pedômetros sobre os fatores de risco metabólicos de crianças obesas, encontrando discretas alterações nas variáveis antropométricas, contudo, quando analisados os fatores de risco metabólicos, foi possível observar uma redução significativa nos valores de insulina e HOMA IR no grupo pedômetro.

A eficácia da utilização de pedômetros para o aumento da atividade física de crianças obesas já foi comprovada em diversos estudos 14,15,22,23. Entretanto, assim como no presente estudo, a maioria das investigações não identificaram mudanças significativas nos indicadores de adiposida$\mathrm{de}^{23-25}$. O tratamento da obesidade somente pela mudança na prática de atividade física pode não ser suficiente para promover diminuições

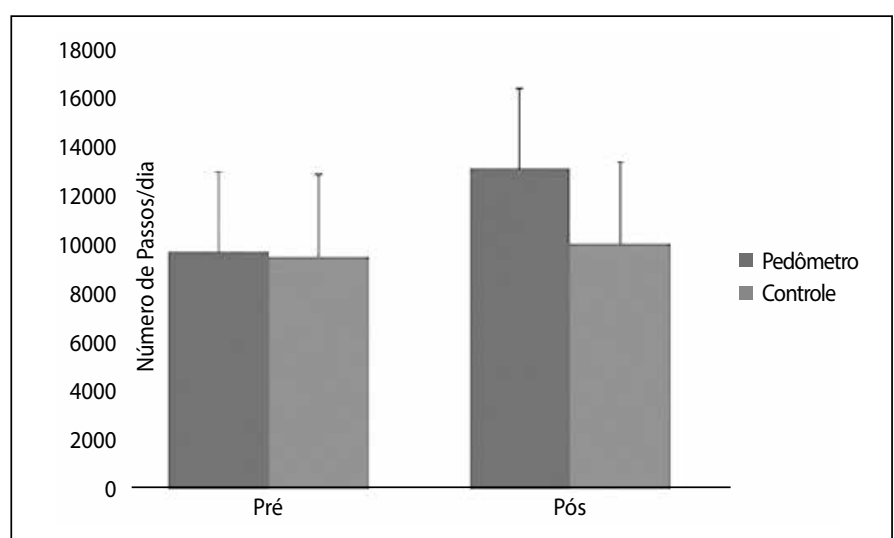

Figura 2. Comparação intra e entre grupos na média do número de passos/dia de uma intervenção de 12 semanas de atividade física utilizando pedômetros. Os dados estão expressos em média e DP.

* Diferença intra grupo em relaçăo ao momento pré $(p<0,05)$; \# Diferença inter grupo no momento pós $(p<0,05)$.

Tabela 1. Características antropométricas de uma intervenção de 12 semanas de atividade física utilizando pedômetros em crianças obesas.

\begin{tabular}{c|c|c|c|c}
\hline \multirow{2}{*}{ Variáveis } & \multicolumn{2}{|c|}{ Pedômetro $(\mathbf{n = 1 0 )}$} & \multicolumn{2}{c}{ Controle $(\mathbf{n}=9)$} \\
\cline { 2 - 5 } & Pré & Pós & Pré & Pós \\
\hline Estatura $(\mathrm{cm})$ & $145,75(5,28)$ & $147,00(5,90)^{*}$ & $148,33(3,74)$ & $149,61(4,18)^{*}$ \\
\hline Massa Corporal $(\mathrm{kg})$ & $57,45(10,42)$ & $57,58(9,83)$ & $56,40(8,28)$ & $58,72(9,34)^{*}$ \\
\hline IMC $\left(\mathrm{kg} / \mathrm{m}^{2}\right)$ & $26,95(3,99)$ & $26,54(3,60)$ & $25,60(3,43)$ & $26,26(4,07)^{*}$ \\
\hline $\mathrm{CC}(\mathrm{cm})$ & $80,95(8,20)$ & $82,90(7,30)$ & $83,83(12,20)$ & $83,22(11,82)$ \\
\hline
\end{tabular}

Os dados estão expressos em média (desvio padrão); ${ }^{*}$ Diferença intra grupo em relaçăo ao momento pré $(p<0,05)$; IMC:Índice de Massa Corporal; CC: circunferência da cintura.

Tabela 2. Comparação intra e inter grupos nos fatores de risco metabólicos de uma intervenção de 12 semanas de atividade física utilizando pedômetros em crianças obesas.

\begin{tabular}{c|c|c|c|c}
\hline \multirow{2}{*}{ Variáveis } & \multicolumn{2}{|c|}{ Pedômetro (n=10) } & \multicolumn{2}{c}{ Controle (n=9) } \\
\cline { 2 - 5 } & Pré & Pós & Pré & Pós \\
\hline \multirow{2}{*}{ PAS (mm.hg) } & $\begin{array}{c}125,00 \\
(13,54)\end{array}$ & $\begin{array}{c}118,00 \\
(16,19)\end{array}$ & $\begin{array}{c}121,33 \\
(16,15)\end{array}$ & $\begin{array}{c}114,44 \\
(11,30)\end{array}$ \\
\hline PAD (mm.hg) & $82,00(11,35)$ & $74,00(11,73)$ & $78,56(7,82)$ & $73,33(13,22)$ \\
\hline \multirow{2}{*}{ Colesterol total (mg.dl) } & 129,90 & 142,43 & 146,78 & 156,44 \\
& $(28,60)$ & $(19,33)$ & $(39,54)$ & $(24,65)$ \\
\hline \multirow{2}{*}{ Triglicerídeos (mg.dl) } & 104,40 & $91,54(17,89)$ & 140,00 & 130,56 \\
& $(42,64)$ & $(70,13)$ & $(63,33)$ \\
\hline HDL-C (mg.dl) & $48,30(11,56)$ & $48,89(10,66)$ & $50,56(13,04)$ & $50,33(8,52)$ \\
\hline Glicose (mg.ml) & $92,10(8,67) \#$ & $85,97(9,04)$ & $80,55(13,47)$ & $82,77(7,49)$ \\
\hline Insulina ( $\mu$ ul.ml) & $12,21(8,53)$ & $7,26(4,70)^{*}$ & $11,40(6,64)$ & $8,50(5,83)$ \\
\hline HOMA IR & $2,76(1,93)$ & $1,49(0,93)^{*}$ & $2,27(1,24)$ & $1,74(1,15)$ \\
\hline
\end{tabular}

Os dados estão expressos em média (desvio padrão); *Diferença significativa intra grupo em relação ao momento pré. " Diferença inter grupos no momento pré $(p<0,05)$; HOMA IR = índice de homeostase. 
significativas nos indicadores de adiposidade em investigações com período relativamente curto de tempo ${ }^{25}$, contudo, vale ressaltar que não houve aumento da massa corporal no grupo pedômetro durante este período como ocorrido no grupo controle.

Nesta investigação, a intervenção de atividade física proposta não modificou significativamente os valores de pressão arterial das crianças. Esses resultados corroboram com os apresentados por Conwell et al. ${ }^{26} \mathrm{e}$ Buonani et al. ${ }^{27}$, nos quais não foram observadas alterações na PAS e PAD após um período relativamente curto de intervenção em crianças obesas.

Com relação aos fatores de risco metabólicos na população pediátrica obesa, Conwell et al. ${ }^{26}$, encontraram resultados semelhantes aos apresentados por esta investigação, nos quais 12 semanas de intervenção com atividade física utilizando pedômetros induziram mudanças significativas no metabolismo glicêmico. Ademais, de acordo com a revisão sistemática realizada por Ho et al. ${ }^{28}$, estudos de intervenção visando a melhora do estilo de vida em crianças obesas, observaram reduções significantes nos valores de insulina e HOMA IR, corroborando com os nossos achados.

As concentrações elevadas de insulina plasmática e HOMA IR induzidas pela obesidade podem ser consideradas um sinal de alerta para o desenvolvimento do diabetes mellitus tipo 2 e de outras alterações metabólicas relacionadas ${ }^{29}$. Dentre os principais mecanismos sugeridos pela literatura, destaca-se a baixa reatividade das células-beta pancreáticas em relação as concentrações elevadas de glicose e a diminuição no número ou ativação dos receptores de insulina das células-alvo que tornam a insulina circulante menos efetiva em sua função de transporte de glicose até as células ${ }^{30}$. Nesse contexto, a promoção de atividade física por meio do estabelecimento de metas utilizando pedômetros pode colaborar para a prevenção do desenvolvimento de morbidades relacionadas ao metabolismo glicêmico ao longo da vida.

É oportuno ressaltar que esta investigação apresenta algumas limitações que merecem ser mencionadas como a falta do controle nutricional e apoio psicológico, bem como a dependência do comprometimento dos pais e das crianças quanto ao cumprimento das metas estabelecidas. Entretanto, o presente estudo demonstrou que 12 semanas de uma intervenção de atividade física utilizando pedômetros diminuíram as concentrações de insulina e HOMA IR de crianças obesas independentemente da perda de peso. Nessa perspectiva, intervenções multidisciplinares que contemplam a promoção da atividade física, controle nutricional e suporte psicológico devem ser realizadas visando a aquisição de conhecimentos relacionados à saúde a fim de propiciar melhora na qualidade de vida e uma possível mudança de comportamento na população pediátrica.

\section{CONCLUSÃO}

A presente intervenção de atividade física a partir do estabelecimento de metas utilizando pedômetros reduziu significativamente as concentrações de insulina e HOMA IR em crianças obesas independentemente da perda de peso. Assim, novas pesquisas devem ser conduzidas para verificar o impacto da promoção de atividade física por meio de pedômetros na prevenção e/ou tratamento dos fatores de risco para as doenças crônicas não transmissíveis em indivíduos jovens.

$\overline{\text { Todos os autores declararam não haver qualquer potencial conflito }}$ de interesses referente a este artigo.

CONTRIBUIÇÕES DOS AUTORES: Cada autor contribuiu individual e significativamente para o desenvolvimento do manuscrito. WFF (0000-0003-3407-6372)*, JPF (0000-0001-6340-3518)* e ASN (0000-0003-2931-943X)* foram os principais contribuintes na redação do manuscrito. RCC (0000-0002-7633-9719)*, CFS (00000001-8741-4051)*, RGME (0000-0002-0040-8212)* e GCS (0000-0003-1593-4546)* realizaram a intervenção com pedômetro, acompanharam os alunos e coletaram os dados. WFF e ASN avaliaram os dados da análise estatística. WFF, JPF, GCS e ASN, realizaram a pesquisa bibliográfica e a revisão do manuscrito. ${ }^{*} \mathrm{ORCID}$ (Open Researcher and Contributor ID).

\section{REFERÊNCIAS}

1. Abdullah A, Amin FA, Stoelwinder J, Tanamas SK, Wolfe R, Barendregt J, et al. Estimating the risk of cardiovascular disease using an obese-years metric. BMJ Open. 2014;4(9):1-10.

2. Toselli S, Gualdi-Russo E, Boulos DNK, Anwar WA, Lakhoua C, Jaouadi I, et al. Prevalence of overweight and obesity in adults from North Africa. Eur J Public Health. 2014;(Suppl 1):31-9.

3. Monteiro CA, Conde WL, Popkin BM. Income-specific trends in obesity in Brazil: 1975-2003. Am J Public Health. 2007;97(10):1808-12.

4. Gallus S, Lugo A, Murisic B, Bosetti C, Boffetta P, La Vecchia C. Overweight and obesity in 16 European countries. Eur J Nutr. 2015;54(5):679-89.

5. MCAllister EJ, Dhurandhar NV, Keith SW, Aronne LJ, Barger J, Baskin M, et al. Ten putative contributors to the obesity epydemic. Crit Rev Food Sci Nutr. 2009;49(10):868-913.

6. United Nations Children's Fund - UNICEF. The state of the world's children 2011 - Adolescence: an age of opportunity. New York: United Nations Children's Fund;2011. p. 34

7. Janssen I, Leblanc AG. Systematic review of the health benefits of physical activity and fitness in school-aged children and youth. Int J Behav Nutr Phys Act. 2010;7:40.

8. Bielemann RM, Domingues MR, Horta BL, Gigante DP. Physical activity from adolescence to young adulthood and bone mineral density in young adults from the 1982 Pelotas (Brazil) Birth Cohort. Prev Med (Baltim). 2014;62:201-7.

9. Bezerra Alves JG, Montenegro FMU, Oliveira F, Alves R V. Prática de esportes durante a adolescência e atividade física de lazer na vida adulta. Rev Bras Med do Esporte. 2005;11(5):291-4.

10. World Health Organization. Global Recommendations on Physical Activity for Health. Genebra:WHO;2010 p. $1-60$

11. Barros SSH, Lopes A da S, Barros MVG. Prevalência de baixo nivel de atividade física em crianças pré-escolares. Rev bras cineantropom e desempenho hum. 2012;14(4):390-400.

12. Moraes AC, Fernandes CA, Elias RG, Nakashima AT, Reichert FF, Falcão MC. Prevalência de inatividade física e fatores associados em adolescentes. Rev Assoc Med Bras. 2009;55(5):523-8.

13. Adams MA, Caparosa S, Thompson S, Norman GJ. Translating physical activity recommendations for overweight adolescents to steps per day. Am J Prev Med. 2009;37(2):137-40.

14. Lubans DR, Morgan PJ, Callister R, Collins CE. Effects of integrating pedometers, parental materials, and E-mail support within an extracurricular school sport intervention. J Adolesc Health. 2009;44(2):176-83.

15. Bravata DM, Smith-Spangler C, Sundaram V, Gienger AL, Lin N, Lewis R, et al. Using pedometers to increase physical activity and improve health: a systematic review. JAMA. 2007;298(19):2296-304
16. Manley D, Cowan P, Graff C, Perlow M, Rice P, Richey P, et al. Self-efficacy, physical activity, and aerobic fitness in middle school children: examination of a pedometer intervention program. J Pediatr Nurs. 2014;29(3):228-37.

17. Kuczmarski RJ, Ogden CL, Guo SS, et al. 2000 CDC growth charts for the United States: Methods and development. National Center for Health Statistics. Vital Health Stat 11(246). 2002.

18. Tudor-Locke C, Hatano Y, Pangrazi RP, Kang M. Revisiting "'How many steps are enough?" Med Sci Sports Exerc. 2008:40(57):S537-43.

19. 19. Sociedade Brasileira de Cardiologia;Sociedade Brasileira de Hipertensao;Sociedade Brasileira de Nefrologia. VI Diretrizes brasileiras de hipertensão. Arq Bras Cardiol. 2010;95(Suppl 1):1-51.

20. Oliveira PO, Souza MLA, Lima MDA. Indice HOMA (homeostasis model assessment) na pratica clínica: uma revisão. J Bras Patol e Med Lab. 2005;41(4):237-43.

21. Lubans D, Morgan P. Evaluation of an extra-curricular school sport programme promoting lifestyle and lifetime activity for adolescents. J Sports Sci. 2008;26(5):519-29.

22. Lubans DR, Morgan PJ, Tudor-Locke C. A systematic review of studies using pedometers to promote physical activity among youth. Prev Med (Baltim). 2009;48(4):307-15.

23. Berry D, Savoye M, Melkus G, Grey M. An intervention for multiethnic obese parents and overweight children. Appl Nurs Res. 2007;20(2):63-71.

24. Schofield L, Mummery WK, Schofield G. Effects of a controlled pedometer-intervention trial for low-active adolescent girls. Med Sci Sports Exerc. 2005;37(8):1414-20.

25. Richardson CR, Newton TL, Abraham JJ, Sen A, Jimbo M, Swartz AM. A meta-analysis of pedometer-based walking interventions and weight loss. Ann Fam Med. 2008;6(1):69-77.

26. Conwell LS, Trost SG, Spence L, Brown WJ, Batch JA. The feasibility of a home-based moderate-intensity physical activity intervention in obese children and adolescents. Br J Sports Med. 2010;44(4):250-5.

27. Buonani C, Fernandes RA, Silveira LS, Bastos KN, Monteiro PA, Viotto Filho I, et al. Prevenção da síndrome metabólica em crianças obesas: uma proposta de intervenção. Rev Paul Pediatr. 2011;29(2):186-92.

28. Ho M, Garnett SP, Baur L, Burrows T, Stewart L, Neve M, et al. Effectiveness of lifestyle interventions in child obesity: systematic review with meta-analysis. Pediatrics. 2012;130(6):e1647-71.

29. Stumvoll M, Goldstein BJ, van Haeften TW. Type 2 diabetes: principles of pathogenesis and therapy. Lancet. 2005;365(9467):1333-46.

30. Nolan CJ, Damm P, Prentki M. Type 2 diabetes across generations: from pathophysiology to prevention and management. Lancet. 2011;378(9786):169-81. 